\title{
Clear mortality gap caused by graft macrosteatosis in Chinese patients after cadaveric liver transplantation
}

\author{
Zhengtao Liu ${ }^{1,2,3}$, Wenchao Wang ${ }^{1,2 \#}$, Li Zhuang ${ }^{4 \#}$, Jingfeng Liu ${ }^{4}$, Shuping Que ${ }^{5,6}$, Dan Zhu ${ }^{4}$, Linfang Dong ${ }^{4}$, \\ Jian Yu ${ }^{1}$, Lin Zhou ${ }^{1,2,3}$, Shusen Zheng ${ }^{1,2,3,4}$ \\ ${ }^{1}$ Division of Hepatobiliary and Pancreatic Surgery, Department of Surgery, First Affiliated Hospital, School of Medicine, Zhejiang University, \\ Hangzhou, China; ${ }^{2}$ NHC Key Laboratory of Combined Multi-Organ Transplantation, Key Laboratory of the Diagnosis and Treatment of \\ Organ Transplantation, CAMS, First Affiliated Hospital, School of Medicine, Zhejiang University, Hangzhou, China; ${ }^{3}$ Key Laboratory of Organ \\ Transplantation of Zhejiang Province, First Affiliated Hospital, School of Medicine, Zhejiang University, Hangzhou , China; ${ }^{4}$ Shulan (Hangzhou) \\ Hospital Affiliated to Zhejiang Shuren University Shulan International Medical College, Hangzhou, China; ${ }^{5}$ Science for Life Laboratory, KTH - \\ Royal Institute of Technology, SE-171 21, Stockholm, Sweden; ${ }^{6}$ Dingxiang Clinics, Hangzhou, China \\ Contributions: (I) Conception and design: Z Liu, L Zhou, S Zheng; (II) Administrative support: None; (III) Provision of study materials or patients: \\ None; (IV) Collection and assembly of data: Z Liu, W Wang, L Zhuang, S Que, J Yu; (V) Data analysis and interpretation: Z Liu, W Wang, \\ L Zhuang, S Que, J Yu; (VI) Manuscript writing: All authors; (VII) Final approval of manuscript: All authors. \\ "These authors contributed equally to this work. \\ Correspondence to: Shusen Zheng. Division of Hepatobiliary and Pancreatic Surgery, Department of Surgery, First Affiliated Hospital, School of \\ Medicine, Zhejiang University, Hangzhou 310003, China. Email: shusenzheng@zju.edu.cn.
}

\begin{abstract}
Background: Liver transplantation (LT) is one of the most effective surgical treatment for patients with end-stage liver disease. Steatosis is a contributor for inferior graft quality. But its impact and safety on transplantation was less assessed in Chinese patients.

Methods: Graft steatosis and related information involved in recipients, donors and surgical procedures were retrospectively collected from 239 patients.

Results: Donor macrosteatosis (MaS) caused about 2.14 and 2.80 folds of increment on patient and graft mortality. Dose-response analysis revealed prominent risk of grafts on overall patient/organ mortality when MaS content exceeded $10 \%(\mathrm{P}<0.05)$. Noteworthy, deaths were only observed in MaS group when concurrent with extremely higher post-transplant alanine aminotransferase (ALT, 64\%). However, microsteatosis (MiS) grafts didn't affect outcomes after LT. In a cohort of Chinese patients, MaS had comprehensive effects on post-transplant outcomes with relatively lower safety threshold at $10 \%$. Mortality gap caused by MaS grafts was observed in patients with severer ischemia reperfusion injury.

Conclusions: Our study revealled the graft $\mathrm{MaS}$ affected the post-transplant outcomes in lower risk cutoff in Chinese patients. Further study is worthy to validate these results and investigate inner mechanism under the phenomenon.
\end{abstract}

Keywords: Steatosis; prognosis; liver transplantation (LT); dose-response analysis

Submitted Aug 22, 2019. Accepted for publication Nov 21, 2019.

doi: $10.21037 / \mathrm{hbsn} .2019 .12 .02$

View this article at: http://dx.doi.org/10.21037/hbsn.2019.12.02

\section{Introduction}

Until now, liver transplantation (LT) is still one of the most effective therapeutic strategy for most end-stage liver disease. Pathologic steatosis is one of the major causes for declined organ utilization. More than half of allografts were discarded for severer steatosis in some specific regions (1).
Fatty infiltration decreased the tolerance of graft hepatic cells on ischemic-reperfusion injuries in many pathological aspects, which might finally cause primary non-function (PNF) and patient death (2-5). While, many prior clinical trials revealed that the use of grafts with mild to moderate steatosis can be relatively safe with comparable risk of post- 
transplant mortality and complications (6-10). [Reference: (I) the use of fatty liver grafts in modern allocation systems risk assessment by the Balance of Risk (BAR) score; (II) the biopsied donor liver: incorporating macrosteatosis into highrisk donor assessment]. Hence, clinicians are trying to expand the donor pool by maximizing the utilization of marginal grafts like steatotic donor liver on the premise of acceptable post-transplant prognosis. And achievements were observed on decrement of waitlist mortality for this endeavor (11-13). Otherwise, strategies were employed from machine perfusion to pharmacological intervention as approaches to salvage the graft would be discarded before $(14,15)$.

Graft steatosis is a quantitative covariate, which can't be simply used to define the boundary between "absolute" acceptance or unacceptance for LT. "Dose-response" risk-effect model seemed more reasonable to present the interrelationship between donor steatosis and posttransplant outcomes (16). However, the continuous risk of graft steatosis on post-transplant prognosis was less discussed in a fixed cohort before.

LT is a complex systematic engineering. And its quality has comprehensive associations with donor, recipient, graft, surgical characteristics and their interaction $(17,18)$. Therefore, it is important to evaluate the continuous effects of steatosis on post-transplant outcomes in predicative model with uniform adjustment of risk index in a fixed cohort.

Previously, less cohort study was performed in Chinese patients to systematically evaluate the impact of graft steatosis on post-transplant complications and graft/patient mortality. In the context of discrepancy between limited organ supply and increasing demands for LT, it is urgent to have accurate assessment on continuous risk trend of donor steatosis on post-transplant prognosis based on the clinical model from Chinese cohort with adjustment of comprehensive risk profiles.

In current cohort-based study, we separately assessed the impacts of risk cofactors from donor, recipient, graft and surgical aspects on post-transplant complications, graft failure and patient survival. Continuous risks of steatosis on post-transplant outcomes were also evaluated based on clinical model constructed before. Interaction of donor steatosis with other risk covariates in the complex network was also assessed. Predictive nomogram was plotted as reference for marginal graft donation. Our study might help clinicians to better deal with the dilemma between utilization and cancellation of steatotic allografts for LT.

We present the following article in accordance with the STROBE reporting checklist (available at http://dx.doi. org/10.21037/hbsn.2019.12.02).

\section{Methods}

\section{Definition for several concept of complications}

Diagnosis of early allograft dysfunction (EAD) was defined according to the updated criteria designed by NicolauRaducu et al. before (19). To be specific, patients who met the following items simultaneously within the first week after liver transplantation including: (I) ALT $>3,000 \mathrm{IU} / \mathrm{mL}$ or AST $>6,000 \mathrm{IU} / \mathrm{mL}$; (II) total bilirubin (TB) $\geq 10 \mathrm{mg} / \mathrm{dL}$; (III) international normalized ratio (INR) $\geq 1.6$ was diagnosed as EAD (19). And diagnostic criteria of PNF was defined as non-recoverable liver function necessitating emergency retransplantation within the first 72 hours after LT (20).

\section{Enrollment criteria and study population}

This study retrospectively enrolled participants who received LT in Shulan (Hangzhou) Hospital in the period from July 1, 2017 to September 30, 2018. Exclusion criteria were listed as follows: (I) adolescent recipients (aged $<18$ years); (II) donor grafts with absence of histological information; (III) multi-organ transplantation ( $\mathrm{n} \geq 2$ ); (IV) ABO-incompatible transplant cases; (V) Living donor LT.

Informed consents were obtained from each enrolled participant. This study was performed in accordance with the Declaration of Helsinki and approved by the ethical board of Shulan Hospital Affiliated to Zhejiang Shuren University Shulan International Medical College (2019IIT-237).

\section{Data collection and biochemical assay}

Indicators which might affect the post-transplant outcomes were collected for further analysis. And the data collected for evaluating the quality of LT can be categorized into following aspects including donor, recipient, graft and surgery related risk factors.

To be specific, donor/recipient demographic data (age, gender, height, weight); recipient pre-operative viral infection, tumor biomarkers, liver function tests (LFTs) including TB, alanine aminotransferase (ALT), and aspartate aminotransferase (AST), prothrombin time (PT), INR in the first 2 weeks after LT.

Blood loss and transfusion of packed red blood cells (pRBC), prothrombin complex concentrate (PCC), fresh 
frozen plasma (FFP); length of operation, cold ischemia time (CIT), warm ischemia time (WIT) were collected respectively from medical record system of International Hospital of Zhejiang University.

Measurement of LFTs, tumor biomarker (AFP) and electrolyte were assayed by and was assayed by automatic biochemistry analyzer (Abbott i2000). The primary disease of recipients and cause of donor death was diagnosed according to ICD-10 criteria (21). Most information can be obtained from medical record system. Covariates with impact on quality of LT including donor, patient, graft and surgery factors was mainly obtained from medical records of Shulan (Hangzhou) hospital.

\section{Liver biopsy and histological analysis}

Wedge liver biopsy was routinely assayed in all grafts after reperfusion. To be specific, liver samples were infiltrated in $10 \%$ of formalin solution and proceeded to hematoxylin and eosin (H\&E) stained sections followed with standardized protocol. Pathological features and presence of hepatocytes with macro-, micro- or mixed steatosis were evaluated according to criteria defined before (22). And steatotic severity was assessed based on percentage of hepatocytes involved with corresponded features by two experienced pathologists under microscopic observation in doubleblinded manner. According to the distribution of cases using steatotic allografts, patients were classified into five groups according to the steatosis degree of allografts $(\leq 10 \%$, $10-20 \%, \geq 20 \%$ ) in categorical analysis.

\section{Risk scoring system and post-transplant complications}

As major predictive system on post-operative risk for patients with chronic liver disease, model for end-stage liver disease (MELD) score was calculated by formula provided in previous study (23). And the Child-Pugh score was counted based on clinical measures including TB, ALB, PT, ascites and encephalopathy interpreted before (24).

With regard to post-transplant outcomes, graft function was assessed by the peak values of hepatic enzyme in first two weeks after LT. And the short-and long-term prognosis of recipients was assessed by occurrence of EAD and PNF, overall survival and rate of graft failure.

\section{Statistic analysis}

Descriptive data in normal distribution was presented as mean \pm standard deviation (SD) and compared by one-way ANOVA; and data in abnormal distribution was presented as median [inter-quartile range (IQR)] and compared by Mann-Whitney $\mathrm{U}$ test. Comparison on distribution in different groups was performed by chi-square test. Multivariable analysis was performed on dichotomous covariates by binary logistic regression model. Hazard ratio (HR) of factors on patient/graft survival was compared by Cox regression model. And analysis was performed by SPSS software (V20.0).

Analysis on dose-response relationship was conducted by two-stage random effects model (25). Risk curves fitting and linearity test was evaluated by STATA software (release 14) according to method described before (16).

Nomogram was formulated based on the risk from multicovariate analysis to quantitatively predict the integrative risk on post-transplant outcomes. Reliability of nomogram was measured by internal validation test. To be specific, calibration curves were plotted to show consistence between actual probability and predicted outcomes from nomogram. Discriminative performance of nomogram was quantitatively assessed by Harrell's C-index (26). Nomogram and related validation test was conducted by rms package in R software (version 3.6.1) (27).

\section{Results}

\section{Demographic and clinical characteristics of enrolled participants}

Totally, 245 transplant cases in 239 patients (6 recipients received re-transplantation) were enrolled into final analysis after excluding 11 cases who didn't meet the selection criteria (nine cases for absence of information on allograft steatosis and two cases for LT for adolescent patient). All patients received cadaveric organ donation in Hepatobiliary and Pancreatic Surgery Department (HBPD) of Zhejiang University International Hospital in the period from July 1, 2017 to September 30, 2018. All transplant cases were mainly performed by one surgeon (SSZ). And prognosis of patients was followed until March 31, 2019, with median follow-up duration on 314 days [interquartile (IQD) time: 192-402 days].

Characteristics of recipients categorized by allograft steatotic status were listed in Table 1. According to allograft steatosis, patients were categorized into non-steatosis, MaS, $\mathrm{MiS}$ and mixed steatosis group. Around half (50.2\%) of donor allograft suffered the steatosis in different severity. 
Table 1 Clinical characteristics of recipients and operational factors classified by graft histological features

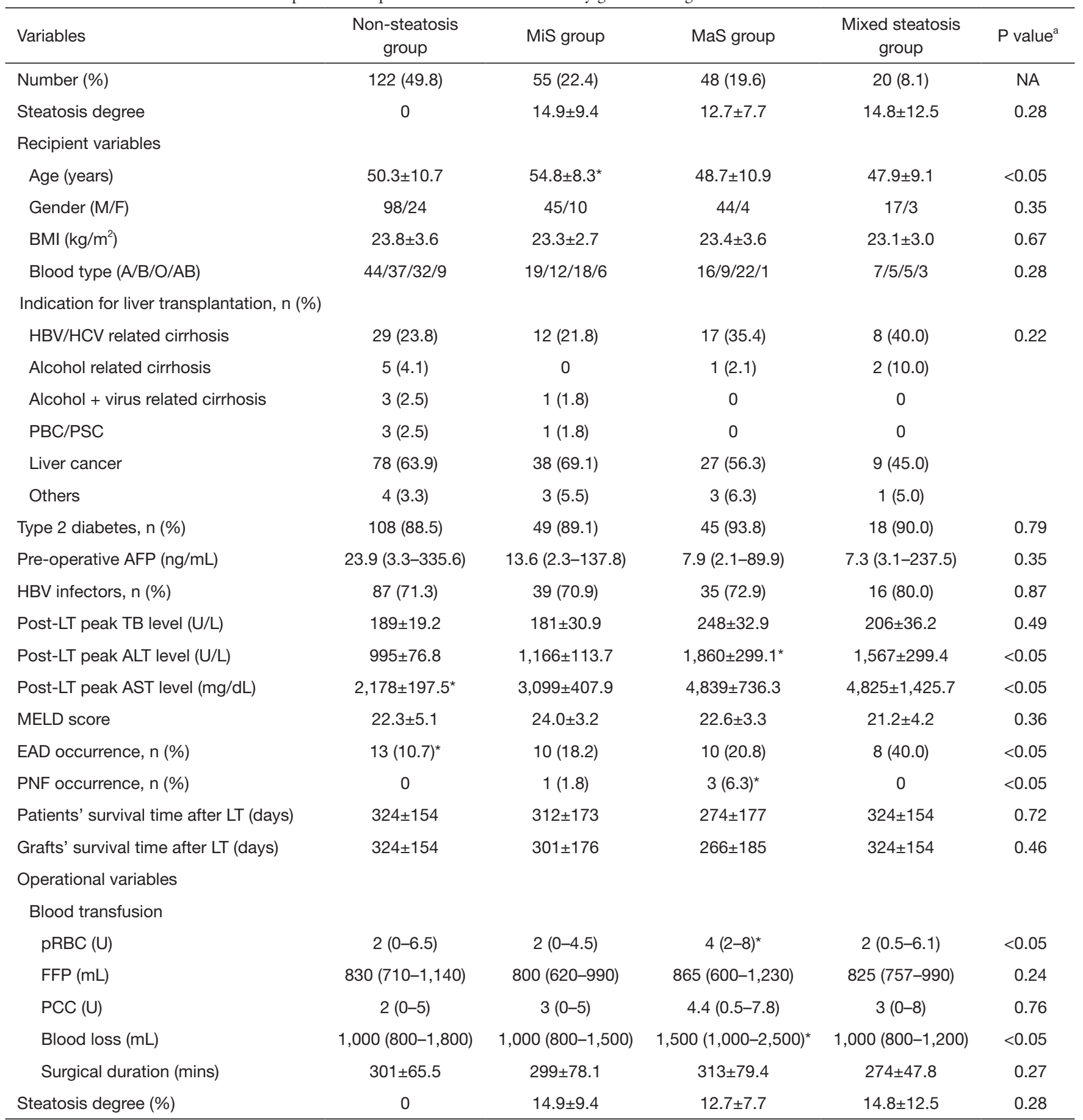

Data was presented in mean \pm SD for data in normal distribution and median (IQR) for data in abnormal distribution. One-way ANOVA test was assayed for normal distributed data; Mann-Whitney $U$ test was assayed for abnormal distributed data; and chi-square test was assayed for patient distribution. * represents the significant difference compared to other groups. ${ }^{\text {a }}$, comparison was performed in groups using grafts with MiS, MaS, and mixed steatosis. AFP, alpha fatoprotein; ALT, alanine aminotransferase; AST, aspertate aminotransferase; $\mathrm{BMI}$, body mass index; EAD, early allograft dysfunction; F, female; FFP, fresh frozen plasma; HBV, hepatitis b virus; HCV, hepatitis $C$ virus; ICU, intensive care unit; LT, liver transplantation; M, male; MELD, Model for End-Stage Liver Disease; NA, not available; TB, total bilirubin; PBC, primary biliary cholangitis; PNF, primary non-function; pRBC, packed red blood cells; PCC, prothrombin complex concentrate; PSC, primary sclerosing cholangitis. 
Table 2 Clinical features of donors and grafts quality classified by donor pathological steatosis severity

\begin{tabular}{|c|c|c|c|c|c|}
\hline Variables & Absent & MiS group & MaS group & Mixed steatosis group & $P$ value $^{a}$ \\
\hline \multicolumn{6}{|l|}{ Donor variables } \\
\hline Age (year) & $44.8 \pm 16.1$ & $35.4 \pm 15.3^{\star}$ & $51.1 \pm 8.0$ & $51.8 \pm 10.9$ & $<0.05$ \\
\hline Gender (M/F) & $56 / 15$ & $24 / 7$ & $20 / 4$ & $11 / 3$ & 0.78 \\
\hline \multicolumn{6}{|l|}{ Pre-donate blood test } \\
\hline Potasium (mmol/L) & $4.2 \pm 0.8$ & $3.8 \pm 1.3$ & $3.9 \pm 0.9$ & $3.7 \pm 1.1$ & 0.21 \\
\hline Sodium (mmol/L) & $144 \pm 9.1$ & $139 \pm 29.4$ & $147 \pm 9.0$ & $145 \pm 9.1$ & 0.34 \\
\hline $\mathrm{ALT}(\mathrm{U} / \mathrm{L})$ & $64.5 \pm 9.9$ & $58.3 \pm 11.4$ & $43.6 \pm 11.9$ & $83.8 \pm 31.9$ & 0.48 \\
\hline BUN (mmol/L) & $10.4 \pm 2.0$ & $8.1 \pm 1.0$ & $7.0 \pm 0.9$ & $10.5 \pm 1.6$ & 0.54 \\
\hline \multicolumn{6}{|l|}{ Graft variables } \\
\hline CIT (min) & $672 \pm 191$ & $698 \pm 176$ & $671 \pm 130$ & $535 \pm 176$ & 0.26 \\
\hline WIT (min) & $15.2 \pm 7.9$ & $15.5 \pm 9.3$ & $15.6 \pm 6.3$ & $17.1 \pm 10.9$ & 0.96 \\
\hline
\end{tabular}

Information was extracted from donors matched to enrolled recipients. Data was presented in mean \pm SD for data in normal distribution and median (IQR) for data in abnormal distribution. ${ }^{\text {a }}$, one-way ANOVA test was assayed for normal distributed data; Mann-Whitney U test was assayed for abnormal distributed data; chi-square test was assayed for patient distribution. * represents the significant difference compared to other groups. M, male; F, female; BMI, body mass index; ALT, alanine aminotransferase; FBG, fasting blood glucose; CR, creatinine; BUN, blood urea nitrogen; CIT, cold ischemia time; WIT, warm ischemia time.

The upper limit for fat content used for LT was 35\% for donor with $\mathrm{MaS}$ or MiS, and 50\% for grafts with mixed steatosis, respectively. Patients across each group was comparable on distribution of gender, blood type, primary liver disease, MELD score, pre-operative body mass index (BMI), $\alpha$-fetoprotein (AFP), operational time, hepatitis B virus $(\mathrm{HBV})$ infection and status of type 2 diabetes $(\mathrm{P}>0.05)$. The mean age of recipients using MiS steatotic allografts was 5.1 years older than patients in non-MiS group (54.8 vs. 49.7 years, $\mathrm{P}<0.05)$. Based on the LFTs results, most patients reached their peak value on liver enzyme $(85.3 \%$ for ALT and $92.2 \%$ for AST), TB (79.5\%), PT (82.8\%) and INR $(83.3 \%)$ in the first or second day after LT (Figure S1). Overall, no difference was observed on the peak values of abovementioned indicators across different post-LT days, except slight decrement on TB value occurred in second post-LT day $(\mathrm{P}<0.05)$. Compared to non-MaS group, the peak value for ALT level was much higher in patients received grafts with $\mathrm{MaS}(1,860$ vs. $1,101 \mathrm{U} / \mathrm{L}, \mathrm{P}<0.05)$. And the peak value for AST level in non-steatosis group was about $55 \%$ lower than the correspond value in $\mathrm{MaS}$ group (2,178 vs. 4,839 U/L, $\mathrm{P}<0.05)$. According to criteria (19) defined before, EAD was occurred in 41 patients (accounting for $16.7 \%$ of whole patients). Compared to patients in steatosis group, EAD occurrence in non-steatosis group was much lower than steatosis group ( $10.7 \%$ vs. $22.8 \%$, $\mathrm{P}<0.05)$. Four cases $(1.6 \%)$ were developed into PNF and allocated for re-transplantation in 3 days after first transplantation. And PNF was occurred only in steatotic groups ( 2 in $\mathrm{MaS}$ and 2 in MiS group, respectively). Cases using MaS allografts had more blood loss (1,500 vs. $1,000 \mathrm{~mL}$ ) and RBC transfusion (4 vs. $2 \mathrm{U}$ ) than non$\mathrm{MaS}$ group (both $\mathrm{P}<0.05$ ). Although relatively shorter length of patient and organ survival was observed in $\mathrm{MaS}$ group, insignificant inter-group difference was presented on follow-up duration across patients received donors with non-steatosis, MaS, MiS and mixed steatosis $(\mathrm{P}>0.05)$.

Matched information was available in 140 donors (accounting for $57.1 \%$ of all LT cases). Categorized by allograft steatosis, donors seemed to be younger in MiS 
Table 3 Impact of steatosis on early allograft dysfunction occurrence after liver transplantation

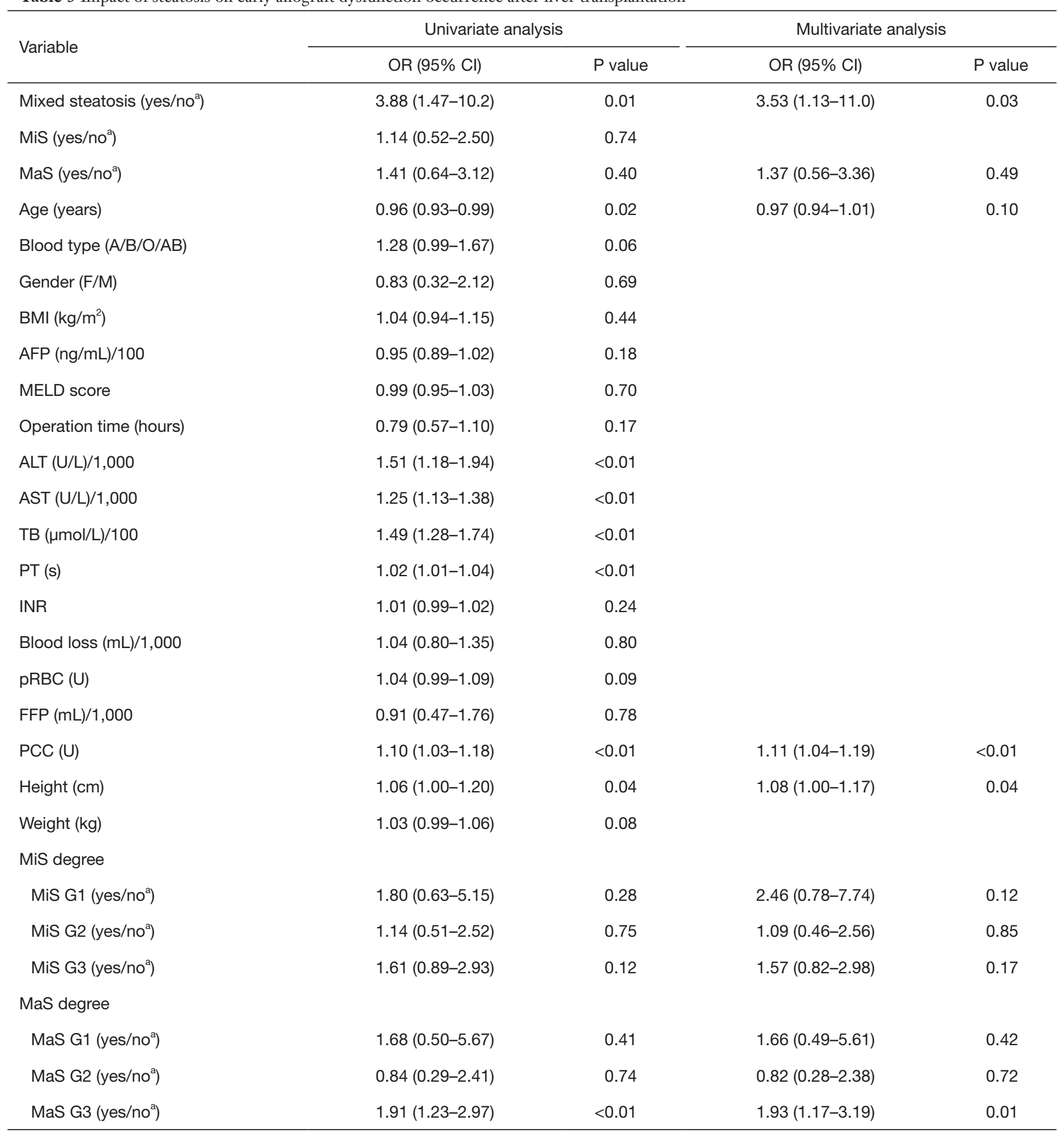

Logistic regression was tested for univariate and multivariate analysis. Factors significantly associated with EAD occurrence were enrolled into multi-covariate logistic regression analysis. " "no" represented the patients received allografts with absence of steatosis (non-steatosis group). G1/G2/G3 respectively represented the steatosis degree (0-10\%], (10-20\%), and [20\%, upper limit]. AFP, alpha fetoprotein; ALT, alanine aminotransferase; AST, aspartate aminotransferase; BMI, body mass index; F, female; INR, international normalized ratio; FFP, fresh frozen plasma; M, male; MaS, macrosteatosis; MELD, model for end-stage liver disease; MiS, microsteatosis; PCC, prothrombin complex concentrate; pRBC, packed red blood cells; PT, prothrombin time; TB, total bilirubin. 

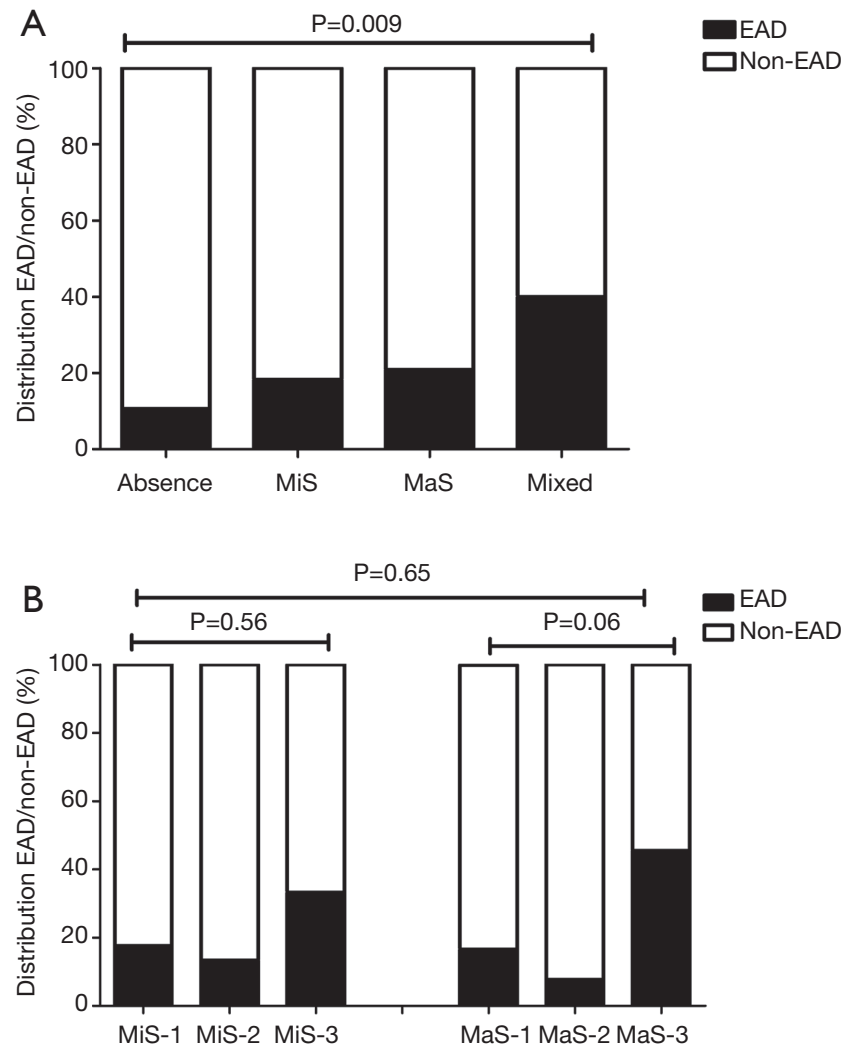

Figure $1 \mathrm{EAD}$ prevalence in patients categorized by steatosis type. (A) EAD prevalence in patients categorized by steatosis type (nonsteatosis, MaS, MiS and mixed steatosis); (B) EAD prevalence in patients categorized by steatotic severity. MiS/MaS-1/2/3 respectively represented the steatosis degree (0-10\%], (10-20\%), and [20\%, upper limit]. Comparison was performed by chi-square test. EAD, early allograft dysfunction; MaS, macrosteatosis; MiS, microsteatosis.

group $(\mathrm{P}<0.05)$. Except that, most indicators that related to graft quality and donor features was comparable in groups categorized by allograft fat content $(\mathrm{P}>0.05$, Table 2$)$.

\section{Risk of donor steatosis on major clinical outcomes}

EAD occurrence and peak levels of ALT/AST were chosen as indicators to evaluate the perioperative effects of LT. Post-operative peak for ALT level was closely correlated to respective peak AST level in whole cohort (pearson coefficient $=0.758, \mathrm{P}<0.05$ ).

In univariate analysis, patients using $\mathrm{MaS}$ grafts had significantly higher post-transplant liver enzyme compared to recipients using non-steatotic allografts (2,072 vs. $844 \mathrm{U} / \mathrm{L}$ for ALT and 4,839 vs. 2,177 U/L for AST level, both $\mathrm{P}<0.05$, Figure S2). Although it seems higher for postoperative peak bilirubin level, donor organ steatosis didn't affect the clinical biochemical indicators including the peak values on TB, PT and INR ( $>>0.05$, Figure S2).

In subsequent stratified analysis, recipient height, allograft with mixed steatosis and transfusion of PCC were potential risk factors for $\mathrm{EAD}$ occurrence $(\mathrm{P}<0.05$, Table 3). EAD prevalence was higher in group using grafts with mixed steatosis (OR $=3.88,95 \%$ CI: 1.47-10.2, Figure $1 A$ and Table 3). EAD in G3 MaS group was significantly higher with about two folds risk than non- $\mathrm{MaS}$ group (OR =1.91, 95\% CI: $1.23-2.97, \mathrm{P}<0.05$, Figure $1 B$ and Table 3). And this effect was still prominent even after adjustment of potential confounders $(\mathrm{P}<0.05$, Table 3). Mixed steatosis might contribute to higher post-transplant AST level $(\mathrm{P}<0.05$, Table S1). And risk of post-transplant AST elevation was higher in G2 and G3 MaS groups, but this trend was overwhelmed after adjustment of post-transplant ALT level (Table S1). Post-transplant ALT level was not associated with stratified donor MaS/MiS severity $(\mathrm{P}>0.05$, Table S2, Table S3).

\section{Risk of allograft steatosis on recipients' prognosis}

The 3-, 6-month and overall patient/organ survival were selected to present the prognosis of patients after LT. The patients were categorized into three groups according to $\mathrm{MaS} / \mathrm{MiS}$ contents $(0<\mathrm{G} 1 \leq 10 \%, 10 \%<\mathrm{G} 2 \leq 20 \%$, G3 $>20 \%$ ). Risk profiles of donor steatosis on posttransplant prognosis was analyzed by combination with other prominent clinical indicators. Compared to non-MaS group, donor $\mathrm{MaS}$ affected the patient survival at borderline significance $(\mathrm{P}=0.07$, Table 4). While this effect became significant on graft survival $(\mathrm{P}<0.05$, Table 5). Allograft $\mathrm{MaS}$ exerted its positive impacts on the whole profiles of patient mortality and organ failure $(\mathrm{P}<0.05)$. To be specific, two to three-folds higher patient death $(\mathrm{OR}=2.14,95 \% \mathrm{CI}$ : $1.06-4.30$, Figure $2 A)$ and graft failure $(\mathrm{OR}=2.80,95 \% \mathrm{CI}$ : $1.51-5.18$, Figure $2 B$ ) was observed in patients using $\mathrm{MaS}$ donors, even after adjusting potential susceptible factors in respective circumstance (Table 4 and Table 5 , all $\mathrm{P}<0.05$ ).

While in stratified analysis, this effect seemed inconsistent followed with increasing MaS severity. Compared to non-steatosis group, G2 MaS induced inferior patient mortality (OR $=2.14,95 \% \mathrm{CI}: 1.06-4.30$, Table 4, Figure $2 A$ ) and graft failure (Table 5, Figure $2 B$ ). While, 
Table 4 Impact of steatosis on patients' survival after liver transplantation

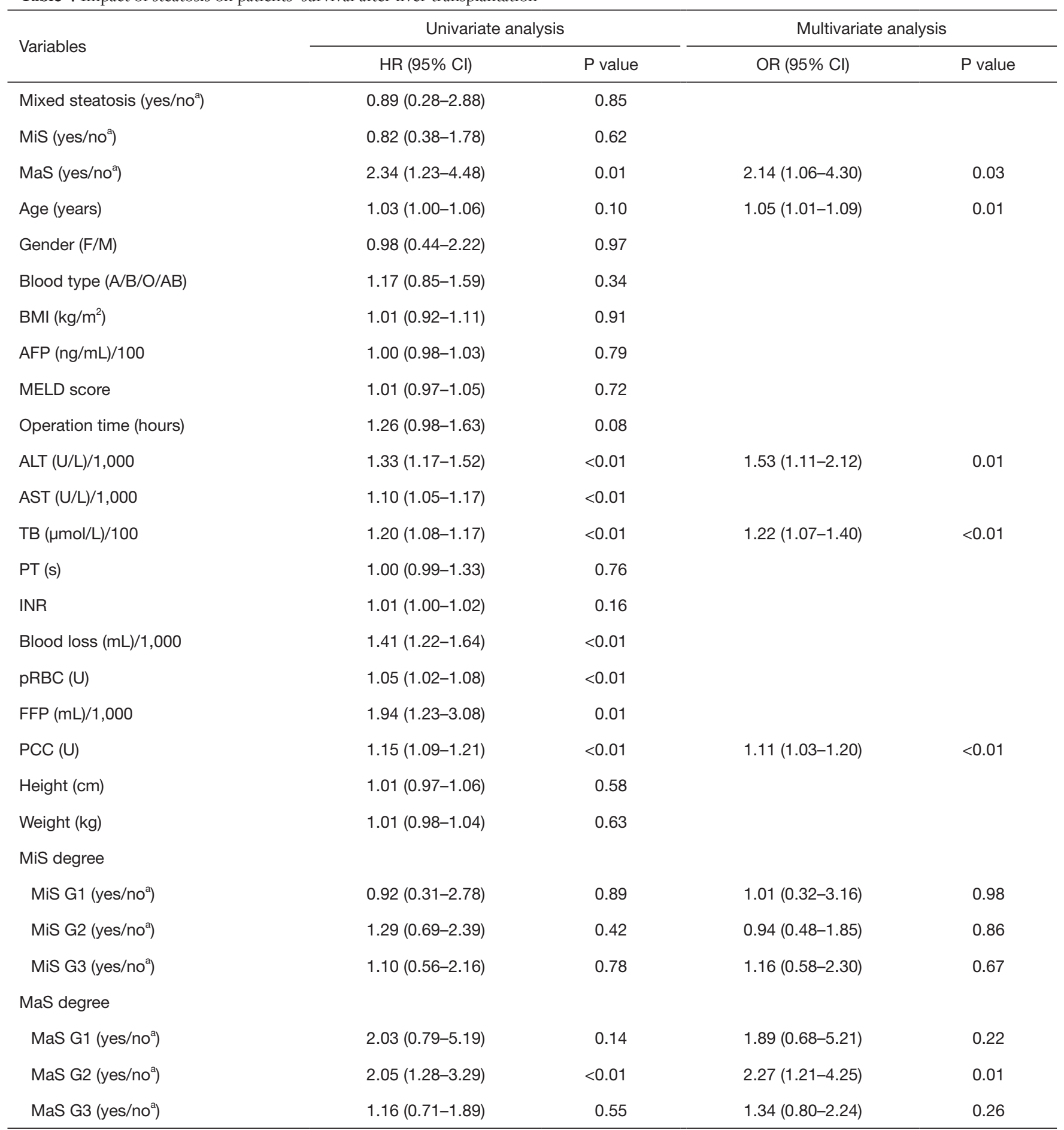

Cox regression model was used for univariate and multivariate analysis. Factors significantly associated with patients' survival (plus age) were enrolled into multi-covariate cox regression analysis. a, "no" represented the patients received allografts with absence of steatosis (non-steatosis group). G1/G2/G3 respectively represented the steatosis degree (0-10\%], (10-20\%), and [20\%, upper limit]. AFP, alpha fetoprotein; ALT, alanine aminotransferase; AST, aspartate aminotransferase; BMI, body mass index; F, female; INR, international normalized ratio; FFP, fresh frozen plasma; M, male; MaS, macrosteatosis; MELD, model for end-stage liver disease; MiS, microsteatosis; PCC, prothrombin complex concentrate; pRBC, packed red blood cells; PT, prothrombin time; TB, total bilirubin. 
Table 5 Impact of steatosis on grafts' failure after liver transplantation

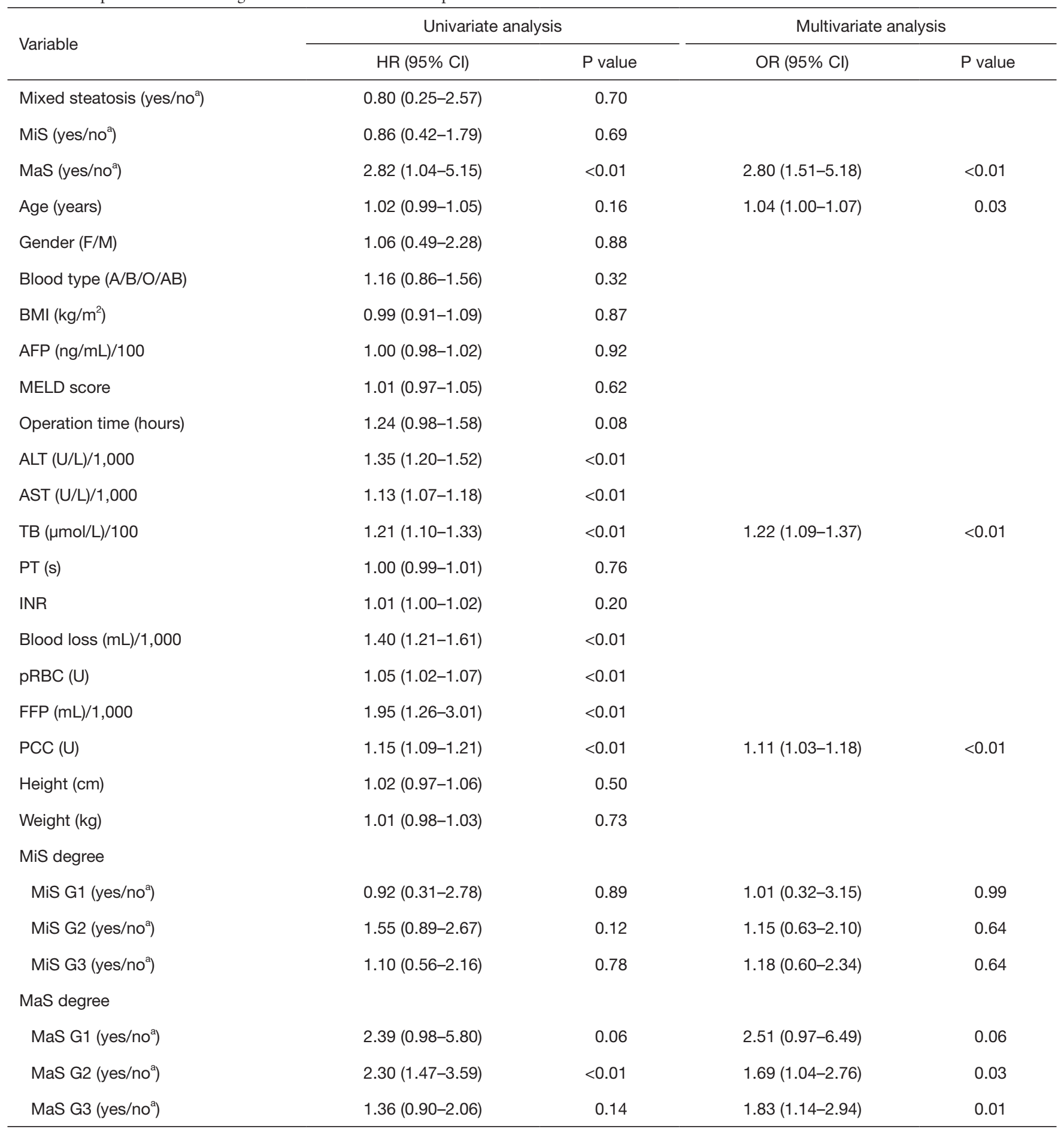

Cox regression model was used for univariate and multivariate analysis. Factors significantly associated with graft failure (plus age) were enrolled into multi-covariate cox regression analysis. ${ }^{a}$, "no" represented the patients received allografts with absence of steatosis (non-steatosis group). G1/G2/G3 respectively represented the steatosis degree (0-10\%], (10-20\%), and [20\%, upper limit]. AFP, alpha fetoprotein; ALT, alanine aminotransferase; AST, aspartate aminotransferase; BMI, body mass index; F, female; INR, international normalized ratio; FFP, fresh frozen plasma; M, male; MaS, macrosteatosis; MELD, model for end-stage liver disease; MiS, microsteatosis; PCC, prothrombin complex concentrate; pRBC, packed red blood cells; PT, prothrombin time; TB, total bilirubin. 

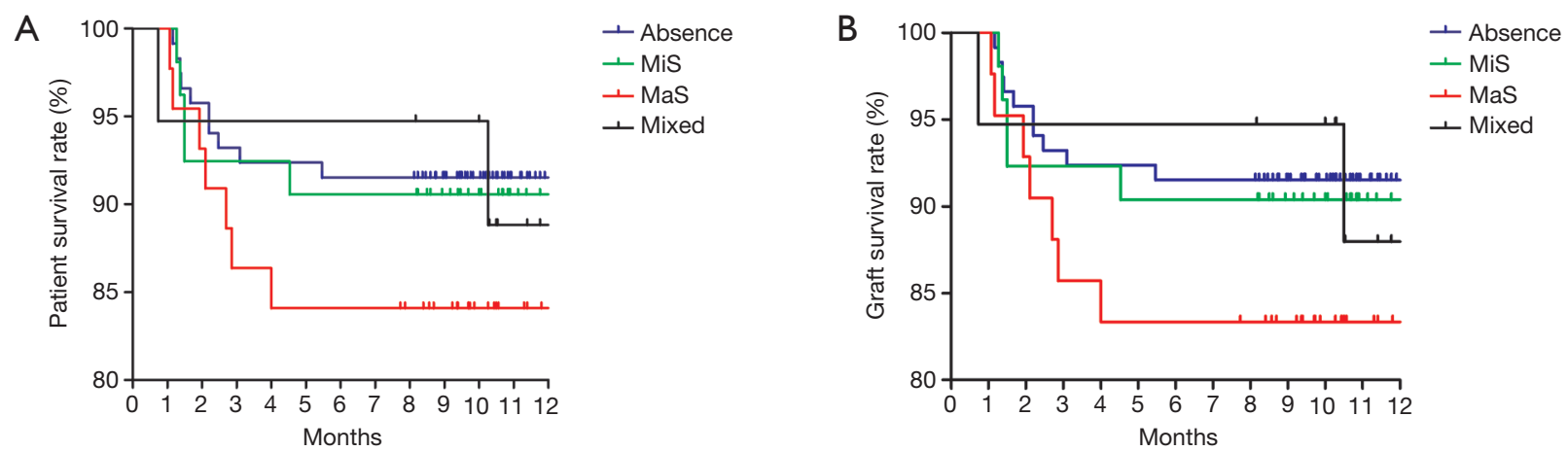

Figure 2 Patient/graft survival rate in patients categorized by graft steatosis type/severity. (A) Patient survival rate categorized by steatosis type; (B) graft survival categorized by steatosis type. Comparison was performed by cox regression model. MaS, macrosteatosis; MiS, microsteatosis.

this positive effect was only presented in G3 MaS group on overall organ failure (OR $=1.83,95 \%$ CI: 1.14-2.94, $\mathrm{P}<0.05$, Figure $2 B$, Table 4). With respect to specific survival time, the risk of G2 MaS was ranged between 1.69 and 2.55 folds on $90-\mathrm{d}$, and $180-\mathrm{d}$ patient/organ mortality, respectively (all $\mathrm{P}<0.05$ ). But $\mathrm{G} 3 \mathrm{MaS}$ lost its significance on risk of patient/organ mortality in respective time points (Tables S4,S5,S6,S7).

As expected, donor MiS or mixed steatosis showed insignificant impact on post-transplant prognosis in multicovariate model ( $\mathrm{P}>0.05$, Table 4, Table 5, and Figure 2). Otherwise, older age, higher post-transplant TB and prothrombin complex transfusion stably affected the overall organ and patient survival by interactive association with donor $\mathrm{MaS}$ in multi-covariate analysis (all $\mathrm{P}<0.05$, Tables 4,5 ).

\section{Does-response relationship between graft steatosis and post-transplant outcomes}

In addition, steatosis degree as a continuous risk on posttransplant outcomes was further quantified by combinative adjustment of prominent clinical factors in prior multicovariate analysis. And risk curve of steatosis on posttransplant outcomes was plotted by instant OR/HR derived in each point (Figure 3 and Figure S3).

Severer $\mathrm{MaS}$ caused significant $\mathrm{EAD}$ increment in linear pattern (Figure $3 A$ ). Compared to non-steatotic cases, about 3.64 folds of higher risk was observed in patients received grafts with $30 \%$ of $\mathrm{MaS}$ content $(\mathrm{OR}=13.7,95 \% \mathrm{CI}$ : 1.74-118.7, $\mathrm{P}<0.05)$. While, the risk trend of organ/patient mortality was not increased accordingly followed with EAD increment (Figure 3). Gentle increment on organ failure was observed in linear manner ( $\mathrm{P}$ for non-linearity $>0.05$ ). And the threshold for $\mathrm{MaS}$ content was approximate $10 \%, 10 \%$ and $15 \%$ for overall, $90-\mathrm{d}$, and $180-\mathrm{d}$ mortality (Figure 3, Table S8).

Interestingly, MaS affected 90-d and overall patient survival in non-linear pattern ( $\mathrm{P}$ for non-linearity test $<0.05$, Figure 3, Table S8). Prominent MaS related risk came into plateau within content between $10 \%$ and $25 \%$, and lost significance in higher $\mathrm{MaS}$ group (>25\%). Linearity was recovered on risk of $180-\mathrm{d}$ patient mortality. And the safety threshold of $\mathrm{MaS}$ was below $15 \%$ for 180 -d patient survival (Figure 3, Table S8).

MiS affected the EAD occurrence and patient/organ mortality in linear pattern. But the trend was insignificant followed with increasing MiS content (all $\mathrm{P}>0.05$, Figure S3, Table S8).

\section{Clear risk stratification in cases using steatotic grafts with simultaneous serious post-transplant liver damage}

Evaluation was performed on inter-relationship across donor steatosis, post-transplant liver function and prognosis. Compared to absent group, patients received $\mathrm{MaS}$ donor tended to have severer liver damage (OR for Q4 ALT $=3.33$, 95\% CI: $1.30-8.54$; and OR for Q4 AST $=4.12$, 95\% CI: $1.65-10.2, \mathrm{P}<0.05$, Figure $4 A$ ). While, this trend was unobvious on TB level (OR for Q4 TB $=2.13$, 95\% CI: $0.83-$ 5.43, $\mathrm{P}=0.11$, Figure 4A). Interestingly, significant increment on peak TB and ALT related mortality was only observed in patients received MiS or non-steatotic grafts $(\mathrm{P}<0.05$, Figure 4). But MaS related mortality was stable and consistent, with independence of TB or ALT classification ( $\mathrm{P}>0.05$, Figure 4$)$. 
A

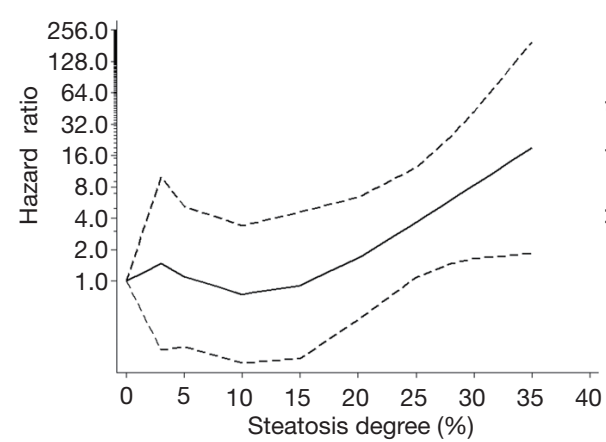

B

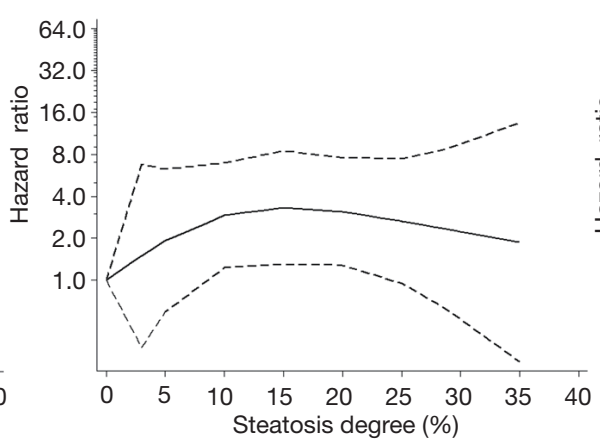

C

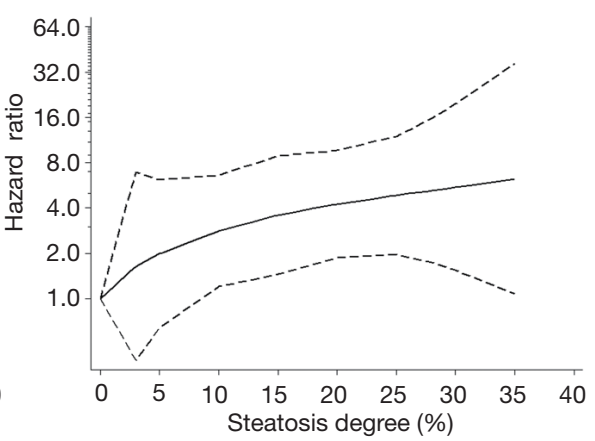

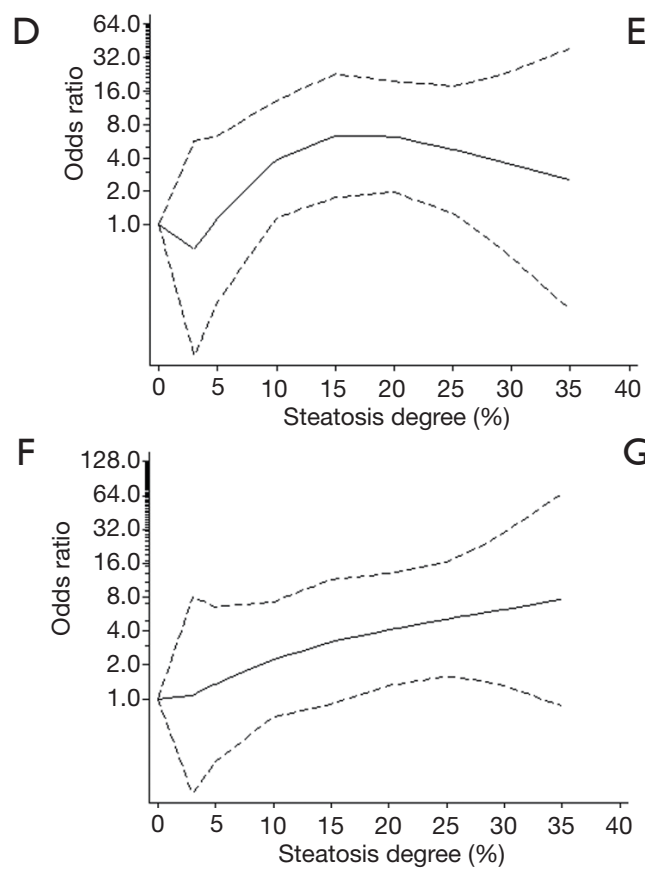

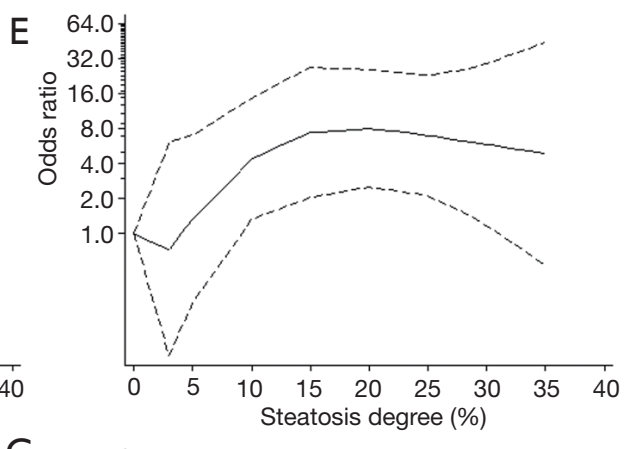

G

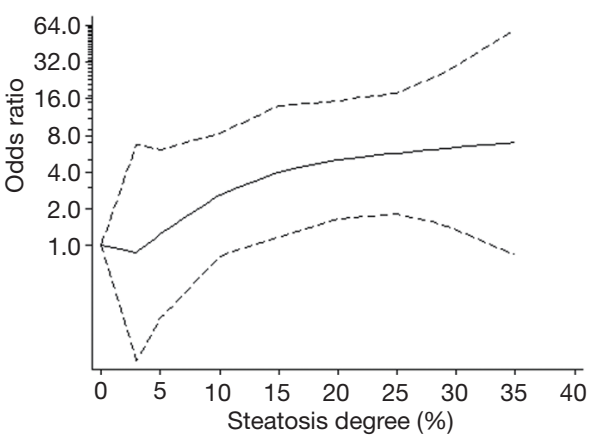

Figure 3 Dose-response risk of increasing MaS degree on post-transplant outcomes. (A) Dose-response risk of increasing MaS degree on EAD occurrence; (B) dose-response risk of increasing MaS degree on patients' mortality; (C) dose-response risk of increasing MaS degree on graft failure; (D) dose-response risk of increasing $\mathrm{MaS}$ degree on 90-day patients' mortality; (E) dose-response risk of increasing $\mathrm{MaS}$ degree on 90-day graft failure; (F) dose-response risk of increasing MaS degree on 180-day patients' mortality; (G) dose-response risk of increasing MaS degree on 180-day graft failure. The black solid and dashed curves represented instant ORs and their respective $95 \%$ CIs for post-transplant outcomes compared to subgroup using allografts without steatosis based on the restricted cubic splines model. CI, confidence interval; EAD, early-allograft dysfunction; MaS, macrovesicular steatosis; OR, odds ratio.

Effects of steatosis were evaluated separately in patients with extremely high ALT $(>2,500 \mathrm{U} / \mathrm{L})$, AST $(>7,000 \mathrm{U} / \mathrm{L})$ or TB $(>400 \mu \mathrm{mol} / \mathrm{L})$ value. Interestingly, death was only occurred in $\mathrm{MaS}$ or mixed group when patients were confined in extremely high ALT level (Figure 4). Much less death was observed in MiS or absent group with extremely high AST level [1 (12.5\%) in MiS group vs. 5 (38.5\%) in $\mathrm{MaS}$ group]. However, this trend was not obvious in patients with extremely high peak TB value. Compared to MiS or non-steatotic group, no more death/graft failure was observed in patients using $\mathrm{MaS}$ allografts (P for distribution $>0.05$, Figure 4).

In addition, death was still frequent $(62.5 \%)$ even in patients without EAD occurrence. MaS effect on post- 


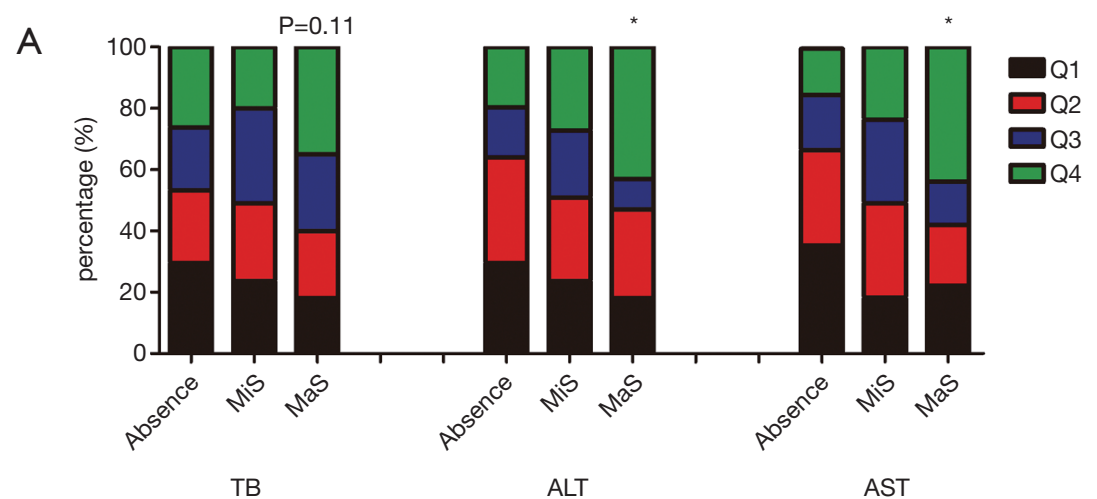

B

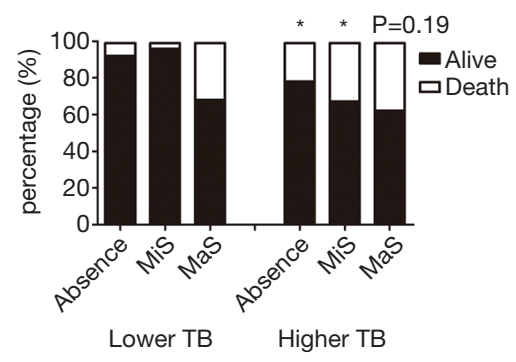

E

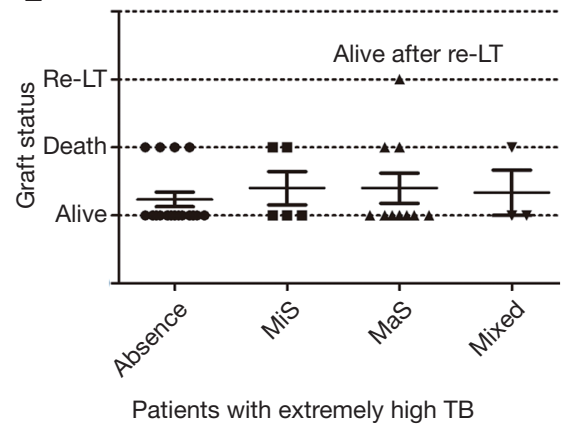

C

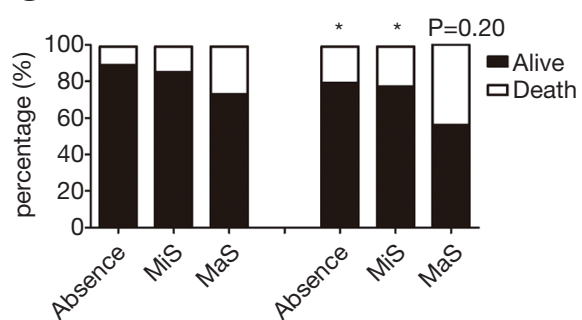

Lower ALT Higher ALT

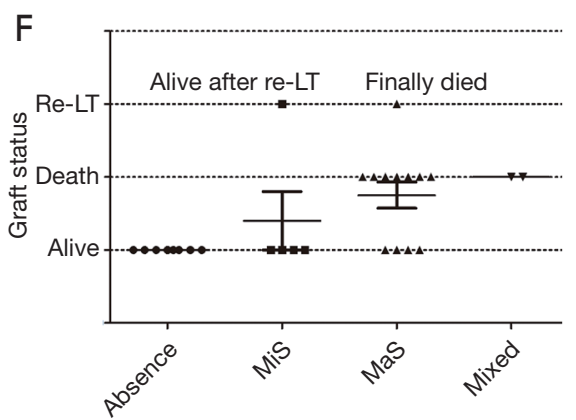

Patients with extremely high ALT

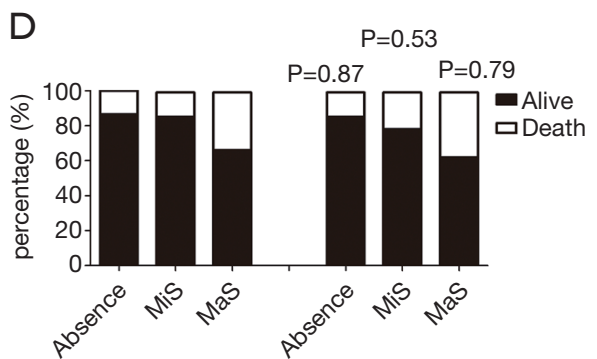

Lower AST Higher AST

G

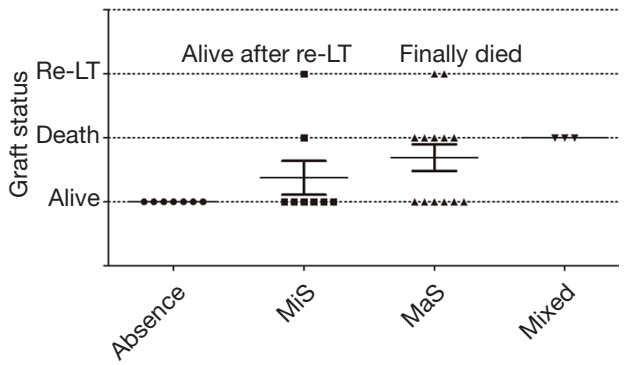

Patients with extremely high AST

Figure 4 Impact of steatosis on stratification of post-transplant LFTs indicators and their integrative effects on prognosis after LT. (A) Stratification of TB, ALT and AST level by quartiles in subgroups categorized by stetosis types, percentage of patients with Q4 ALT/AST was higher in MaS group; (B) mortality stratification in MaS, MiS and absence subgroups categorized by TB level, elevated mortality was presented in MiS and absence group caused by higher TB level; (C) mortality stratification in MaS MiS and absence subgroups categorized by ALT level, elevated mortality was presented in MiS and absence group caused by higher ALT level; (D) mortality stratification in MaS MiS and absence subgroups categorized by AST level, elevated mortality was presented in MiS and absence group caused by higher AST level; (E) stratification of graft status categorized by steatosis type in patients with extremely higher TB value; (F) stratification of graft status categorized by steatosis type in patients with extremely higher ALT value; (G) stratification of graft status categorized by steatosis type in patients with extremely higher ALT value. * represented significant difference in corresponded comparisons $(\mathrm{P}<0.05)$. $\mathrm{P}$ value above the bars represented the significance in corresponded comparisons. ALT, alanine aminotransferase; AST, aspartate aminotransferase; LFTs, liver function tests; LT, liver transplantation; MaS, macro-steatosis; MiS, micro-steatosis; TB, total bilirubin.

transplant prognosis was independent of EAD prevalence in subgroup with extremely high ALT ( $>>0.05$ for distribution, Table S9). Therefore, MaS allografts seemed to have significant impacts on post-transplant aminotransferase but not bilirubin levels. Meanwhile, donor MaS significantly worsen the post-transplant survival in patients experienced severe hepatic damage (presented by extremely high ALT or AST). And the inter-relationship amongst donor MaS, LFTs, 


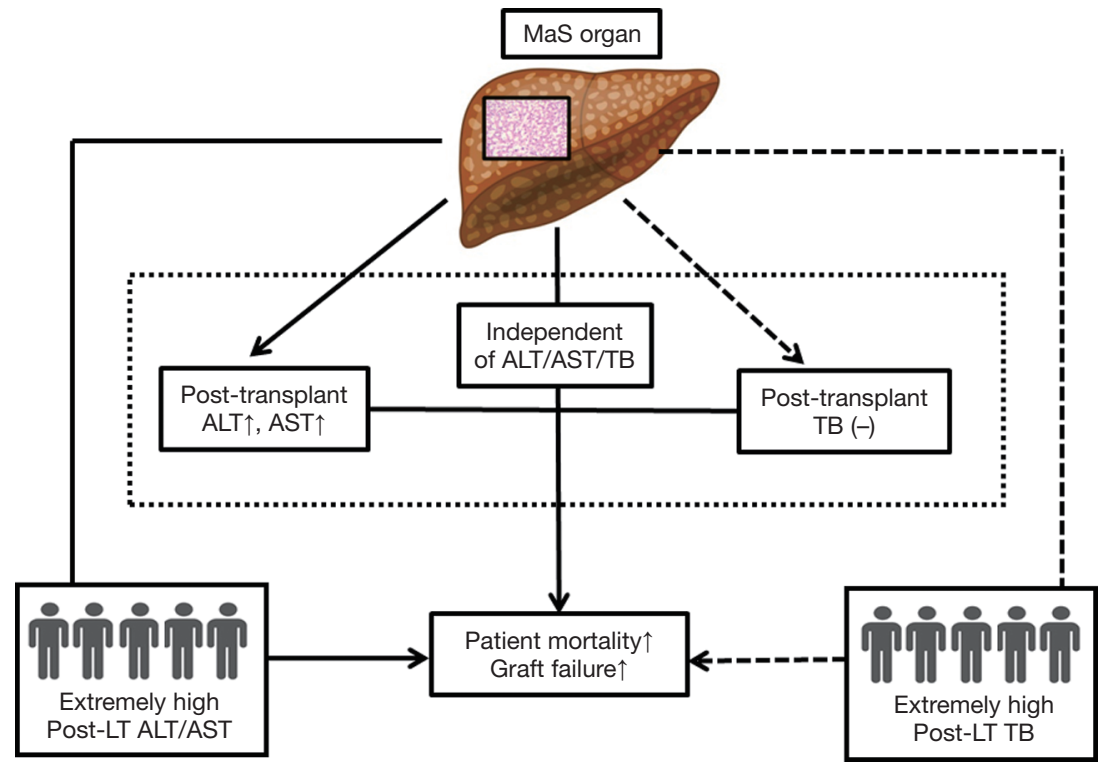

Figure 5 Schematic diagram revealing the complex inter-relationship amongst MaS and post-transplant LFTs and prognosis. Solid arrowed line was represented as positive connection between two covariates (e.g., MaS and post-transplant ALT level); dashed arrowed line was represented as negative connection between two covariates (e.g., MaS and post-transplant TB level). ALT, alanine aminotransferase; AST, aspartate aminotransferase; LT, liver transplantation; MaS, macrosteatosis; TB, total bilirubin.

and post-transplant outcomes were summarized in Figure 5.

\section{Disease outcome of re-transplanted case series and their association with allograft steatosis}

Features of cases received re-transplantation were listed respectively in Table 6. Six patients had re-transplantation in $1-15$ days after first operation for the failure of transplanted grafts. PNF was defined in 4 of these cases $(1.6 \%)$ for shorter interval ( $<72$ hours) across two LT.

Donor MaS seemed to be the major contributor of PNF and related mortality. In our cohort, two of four PNF patients received MaS grafts for first LT. Otherwise, both two death cases after re-transplantation received $\mathrm{MaS}$ organs in the first LT. The only one survivor (case 4) received $\mathrm{MaS}$ related LT might be benefited from his relative younger age (30 years) and less burden of primitive liver disease (non-cancer, absence of $\mathrm{HBV}$ infection). $\mathrm{MaS}$ donor seemed not the absolute contradiction for further transplantation, but the recipient should be highly selective in less serious patients for better prognosis. Unlike the $\mathrm{MaS}$ donors, it seemed to be safer for LT with the use of MiS grafts. Two MiS related PNF cases had favorable prognosis after re-transplantation. And no death was observed in the end of follow-up duration (Table 6).
Correspondingly, trend of LFTs indicators and PT value was presented respectively in peri-operative period from 3 days before LT to 2 weeks after LT. As shown in Figure S4, post-transplant liver transaminase seemed much higher in death cases. The peak AST level was higher in both death cases $(>15,000 \mathrm{U} / \mathrm{L})$ than the remaining alive cases $(<8,000 \mathrm{U} / \mathrm{L})$. However, post-transplant PT and TB value was comparable across individuals, regardless of difference on post-transplant prognosis.-

\section{Individualized risk nomogram of post-transplant outcomes and internal validation test}

Nomograms for prediction of post-transplant prognosis and complications was plotted by incorporation of graft steatosis and other independent clinical factor which was validated in aforementioned risk evaluation. MaS caused severer posttransplant outcomes followed with increasing severity by gradient. Severer MaS (G3) caused significant decrease on post-transplant patient/graft survival. To be specific, the 180-day patient/graft survival was decreased to less than $85 \%$ and around $80 \%$ in patients using severer MaS organs (Figure 6). MiS or mixed allograft steatosis can also predict parts of EAD occurrence, but these effects were limited and inconsistent (Figure 6). C-index for nomogram on EAD 


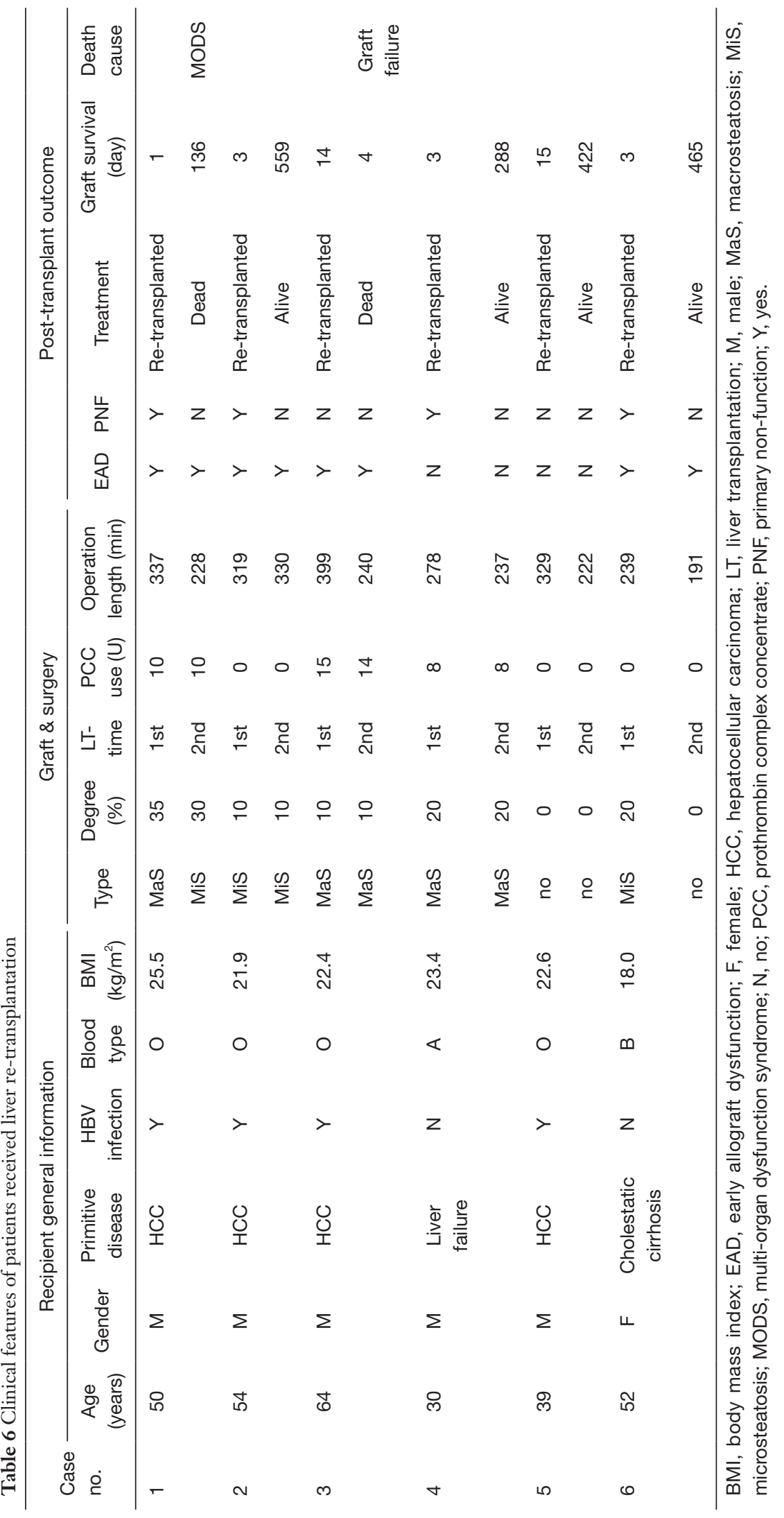


A Points

MaS

MiS

Mixed

Recipient_age

Height

PCC transfusion

Total points

Risk of EAD

B Points

MaS

MiS

Mixed

Peak_TB

PCC transfusion

Recipient_age

Total points

90-day survival

180-day survival

C Points

MaS

MiS

Mixed

Peak_TB

PCC transfusion

Recipient_age

Total points

90-day survival

180-day survival
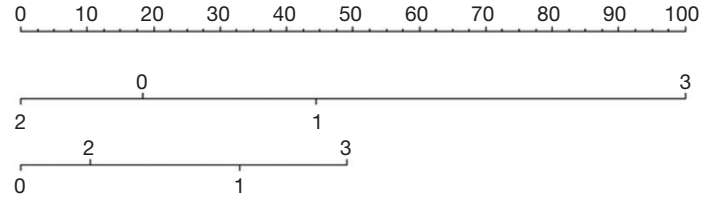

Yes
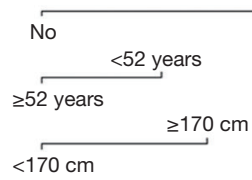

$\geq 2 U$

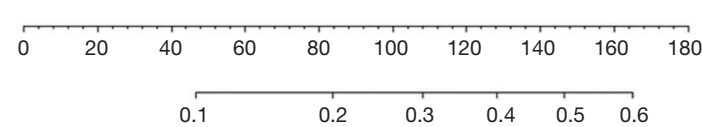

$\begin{array}{lllllllllll}0 & 10 & 20 & 30 & 40 & 50 & 60 & 70 & 80 & 90 & 100\end{array}$
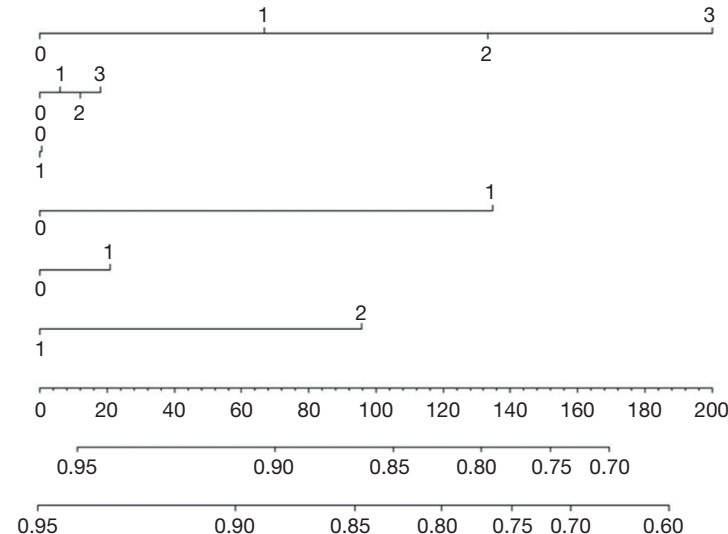

$\begin{array}{lllllllllll}0 & 10 & 20 & 30 & 40 & 50 & 60 & 70 & 80 & 90 & 100\end{array}$

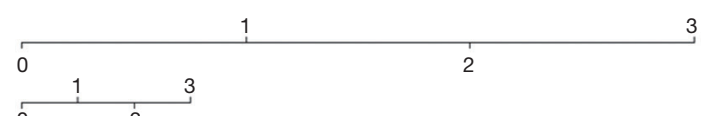

+
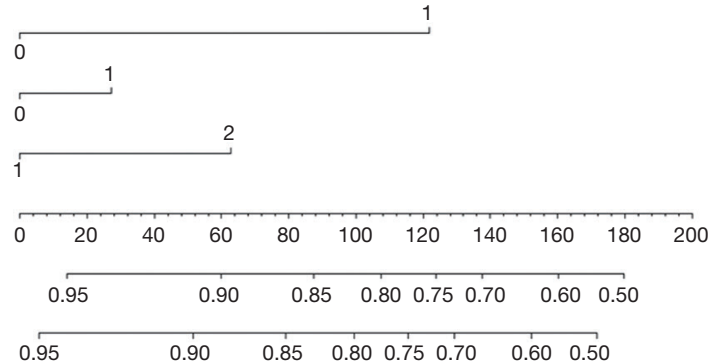

Figure 6 Nomogram for prediction of outcomes in patients after liver transplantation. Risk of incidence is located on the point in each point in each variable axis, and line drawn upwards can determine the points received for each variable. Cumulative risk of individual factors is located on total point axis, and the line drawn downwards can determine the risk of each post-transplant outcomes. (A) Nomogram for prediction of post-transplant EAD occurrence; (B) nomogram for prediction of 90-, 180-day patients' survival after LT; (C) nomogram for prediction of 90-, 180-day grafts' survival after LT. MaS, macrosteatosis; MiS, microsteatosis; PCC, prothrombin complex concentrate; TB, total bilirubin. 
occurrence, patient and graft survival was $0.75(0.73-0.77)$, $0.73(0.71-0.75)$, and $0.73(0.71-0.75)$, respectively. And calibration plot revealed consistent trend between actual and nomogram predicted post-transplant outcomes (Figure S5).

\section{Discussion}

Currently, allograft steatosis is one of the most prominent risk cofactor on inferior post-transplant survival (28). Emerging evidence has revealed that steatosis (especially for $\mathrm{MaS}$ ) was the major reason for potential allograft being discarded for transplantations, affecting $40-60 \%$ of cancelled organ donation $(1,29)$. While, expansion of donor pool is urgently needed by optimizing the utilization of steatotic allografts to meet the demands from increasing patients on waitlist for LT. Meanwhile, concurrence of steatosis with other risk factor was also observed before (28). Therefore, continuous risk profiles of donor steatosis in complex context of specific population should be assessed to keep benefits from liver transplantation on the premise of acceptable post-operational prognosis. In a Chinese cohort from a newly-established transplant center, we found: (I) steatosis was highly prevalent in Chinese donation for LT, affecting around half (48.6\%) of allografts; (II) MaS (but not MiS) amplified the risk of inferior post-transplant prognosis; (III) more complications and inferior prognosis were presented followed with increasing $\mathrm{MaS}$ (but not MiS) severity in dose-response manner; (IV) Chinese recipients seemed more susceptive to $\mathrm{MaS}$ grafts and the safety threshold of $\mathrm{MaS}$ was lowered to $10 \%$; (V) positive impacts of $\mathrm{MaS}$ on post-transplant complications were stable and consistent with independence of peak bilirubin or transaminase level; (VI) MaS graft application in first LT was the main cause of death in patients received retransplantation; (VII) mortality gap was clearly stratified by MaS classification, in patients with serious liver damage.

To the extent of our knowledge, this is a retrospective study with enrollment of largest Chinese patients with focus on building connection between allograft steatosis and post-transplant outcomes. We systematically reviewed the published data (16) and found only one literature (30) assessed the continuous risk of $\mathrm{MaS}$ on post-transplant outcomes in Chinese recipients. In the small sized cohort (70 LT pairs), Li et al. found insignificant impact of MaS on patients' prognosis, but the conclusion seemed inaccurate for inappropriate classification in comparison. Patients using non-steatotic grafts weren't categorized as an independent group which might cause overestimation of baseline data. In our study, obvious risk was presented on overall patient/graft mortality. Categorical comparison revealed insignificant risk for severe MaS organ (>20\%) on patients' survival (Table 4). However, "severity-response" analysis found the risk for $\mathrm{MaS}$ was stable and consistent on patient/organ mortality when MaS exceeded 10\% (Table S8). Interestingly, risk plateau was observed between moderate and severe MaS group, and patient mortality didn't increase linearly followed with MaS severity (Table S8). However, the risk of $\mathrm{MaS}$ grafts still exists on post-LT prognosis for higher organ failure followed with increasing MaS severity in linear pattern (Figure 3, Table S8). Unlike MaS, there seems no obvious connection between MiS allograft and inferior prognosis in categorical comparison and continuous regression model (Figure 3, Table S9) conformed to majority of other studies (6,31-33).

Conformed to prior study (34), severe MaS also increased the post-transplant EAD occurrence in our patient cohort. However, the risk cut-off of MaS on EAD occurrence was around $25 \%$, much higher than expected safety threshold of MaS patient/organ mortality (10\%, Table 3). Otherwise, similar transaminase level was observed in patients grouped by MaS severity. These results indicated MaS organs might have inferior effects even in similar post-transplant recovery. More concerns are needed on MaS organ recipients. Consistent with previous studies $(35,36)$, retransplantation was mainly contributor for PNF/DNF, and MaS was the major culprit for death of retransplanted patients in our study (Table 6). Two re-LT patients received MaS allografts had inferior prognosis, regardless of the quality of second implants, but another relatively younger patient with nonmalignant disease was still alive until the end of followup duration. And this discrepancy implied the importance of recipient selection in utilization of steatotic organ on further LT (37). However, less patients with re-LT in this study and more cases are needed for further validation.

ALT is a sensitive biomarker in detecting and screening liver damage (38). But its clinical meaning and interactions with allograft MaS on prognosis was still unclear. Peak ALT level was considered as a quantitative biomarker to evaluate the extend of ischemia-reperfusion injury (IRI) (39). Knowledge from animal experiment found hepatic steatosis per se might cause severer injury in the same circumstance of ischemia and reperfusion treatment $(5,40)$. In our study, donor MaS seemed to have complex association by interaction with peak liver enzyme and post-transplant prognosis. Organ MaS caused more IRI presented as higher peak ALT in patients after LT. But on the other 
side, $\mathrm{MaS}$ induced inferior prognosis independent of its effects on ALT elevation. In addition, clear mortality gap was observed in subgroup with severer IRI pressure. In patients with extremely higher post-transplant ALT level, disproportionately higher mortality was observed in patients received MaS organ (64\% vs. $0 \%$ for MaS vs. MiS, Figure 4). Similar effect was also observed in group with extremely high AST for its correlation with ALT. Noteworthy, graft loss was highly prevalent in $\mathrm{MaS}$ patients, regardless of EAD occurrence after LT. These data revealed MaS (but not MiS) organ might be more fragile under the exposure of severer IRI. And this effect can last longer than perioperative period. Coincident with our results, scholars found the quality of $\mathrm{MaS}$ donor could be guaranteed on the premise of limited CIT (8). Hence, MaS organ should be treated with more cautions or avoided to be used in patients with high-risk of severer post-transplant IRI.

Except MaS, clinical factors including the intra-operative PCC transfusion and post-transplant peak TB value also affected the prognosis of patients after LT (Table 4 and Table 5). Increased blood product transfusion is usually connected to adverse post-transplant outcomes after LT (41). PCC administration was considered as safe and effective strategy for improved haemostasis in LT (42). But our results found higher patient/organ mortality followed in doseresponse manner with unclear reasons. More concerns should be raised on management of PCC utilization in further clinical practice. In consistence with previous study (42), elevated post-transplant TB value was another stronger indictor on prediction of severer adverse outcomes after LT. However, we didn't find donor $\mathrm{MaS}$ had interactive effects on intra-operative PCC transfusion or post-transplant TB value. After adjustment of these potential confounders, $\mathrm{MaS}$ was still a stable and independent predictor on inferior posttransplant outcomes.

MaS but not MiS might affect recipient post-LT prognosis in our study. The MaS organ seemed to have lower resistance under the same ischemia-reperfusion exposure. However, the reason is unclear and worthy for elucidation (14). Croome et al. summarized potential mechanism with connection between $\mathrm{MaS}$ and liver damage, including impaired sinusoidal blood flow, abnormal mitochondria function, increased neutrophil aggregation and cytokine release (37). Meanwhile, cumulative evidence revealed genetic factors might be involved in graft steatosis and LT $(43,44)$. In addition, machine perfusion (MP) is considered as an effective strategy for recondition of steatotic grafts for LT (29). Integrated multi-omic analysis might help to find out the main culprits covered under the complex network and potential targets for improving the organ quality (45).

In our study, all cases were collected from a newly established LT center, and finished by a senior experienced surgeon (SSZ). Unified standard was adopted on donor/ recipient selection, surgical procedure and perioperative management, which can avoid potential confounders as guarantee and prerequisite for further comparison. Limitations of this study should also be noted as well. First, relatively shorter follow-up in enrolled patients. Second, results of this study need validation from external cohort. Third, potential bias was inevitable for disequilibrium on age distribution and missing information in donors. Forth, previous study found possible stratification in MaS group for varied Liver texture between large-droplet and smalldroplet MaS donors (10). This internal bias might also cause inaccurate results into risk assessment. Fifth, precise MaS cut-off for LT should also be assessed by comparison with similar patients who chose non-transplant therapies, which we didn't perform. But regardless of these limitations, results are still worthy to be reported for its novelty and revelation on further mechanistic investigation.

\section{Conclusions}

In conclusion, we found donor MaS, but MiS positively affected post-transplant prognosis and complication with relatively lower safety threshold (around $10 \%$ ) for LT in Chinese patient cohort. Stable effects were exerted by $\mathrm{MaS}$ on patient death with independence of posttransplant liver damage. MaS related mortality gap was much more prominent in patients with extremely high post-transplant transaminase levels. Allograft $\mathrm{MaS}$ was the major cause of PNF occurrence and patients' death after retransplantation. Further external validation in larger cohort and mechanistic investigation are needed to validate these findings.

\section{Acknowledgments}

Funding: This study is supported by Innovative Research Groups of National Natural Science Foundation of China (81721091), Major program of National Natural Science Foundation of China (91542205), National S\&T Major Project (2017ZX10203205), National Natural 
Science Foundation of China (81902813), Zhejiang International Science and Technology Cooperation Project (2016C04003), Zhejiang Provincial Natural Science Foundation of China (LY18H030002), Zhejiang Medical Association (2019ZYC-A81), International Youth Exchange Programme by China Association for Science and Technology (2019), Tianqing Liver Diseases Research Fund (TQGB20200114), and Open Fund of Key laboratory of High-Incidence-Tumor Prevention \& Treatment (Guangxi Medical University), Ministry of Education.

\section{Footnote}

Reporting Checklist: The authors have completed the STROBE reporting checklist. Available at http://dx.doi. org/10.21037/hbsn.2019.12.02

Data Sharing Statement: Available at http://dx.doi. org/10.21037/hbsn.2019.12.02

Conflicts of Interest: All authors have completed the ICMJE uniform disclosure form (available at http://dx.doi. org/10.21037/hbsn.2019.12.02). The authors have no conflicts of interest to declare.

Ethical Statement: The authors are accountable for all aspects of the work in ensuring that questions related to the accuracy or integrity of any part of the work are appropriately investigated and resolved. Informed consents were obtained from each enrolled participant. This study was performed in accordance with the Declaration of Helsinki and approved by the ethical board of Shulan Hospital Affiliated to Zhejiang Shuren University Shulan International Medical College (2019-IIT-237).

Open Access Statement: This is an Open Access article distributed in accordance with the Creative Commons Attribution-NonCommercial-NoDerivs 4.0 International License (CC BY-NC-ND 4.0), which permits the noncommercial replication and distribution of the article with the strict proviso that no changes or edits are made and the original work is properly cited (including links to both the formal publication through the relevant DOI and the license). See: https://creativecommons.org/licenses/by-nc-nd/4.0/.

\section{References}

1. Moosburner S, Gassner JM, Nösser M, et al. Prevalence of steatosis hepatis in the eurotransplant region: impact on graft acceptance rates. HPB Surg 2018;2018:6094936.

2. Koneru B, Dikdan G. Hepatic steatosis and liver transplantation current clinical and experimental perspectives. Transplantation 2002;73:325-30.

3. Selzner N, Selzner M, Jochum W, et al. Mouse livers with macrosteatosis are more susceptible to normothermic ischemic injury than those with microsteatosis. J Hepatol 2006;44:694-701.

4. Hatsugai K, Ohkohchi N, Fukumori T, et al. Mechanism of primary graft non-function in a rat model for fatty liver transplantation. Transpl Int 2000;13:S583-90.

5. Liss KH, McCommis KS, Chambers KT, et al. The impact of diet-induced hepatic steatosis in a murine model of hepatic ischemia/reperfusion injury. Liver Transpl 2018;24:908-21.

6. Ahmed EA, El-Badry AM, Mocchegiani F, et al. Impact of graft steatosis on postoperative complications after liver transplantation. Surg J (N Y) 2018;4:e188-96.

7. Wong TC, Fung JY, Chok KS, et al. Excellent outcomes of liver transplantation using severely steatotic grafts from brain-dead donors. Liver Transpl 2016;22:226-36.

8. Westerkamp AC, de Boer MT, van den Berg AP, et al. Similar outcome after transplantation of moderate macrovesicular steatotic and nonsteatotic livers when the cold ischemia time is kept very short. Transpl Int 2015;28:319-29.

9. Dutkowski P, Schlegel A, Slankamenac K, et al. The use of fatty liver grafts in modern allocation systems: risk assessment by the balance of risk (BAR) score. Ann Surg 2012;256:861-8.

10. Bidkhori G, Benfeitas R, Klevstig M, et al. Metabolic network-based stratification of hepatocellular carcinoma reveals three distinct tumor subtypes. Proc Natl Acad Sci U S A 2018;115:E11874-83.

11. Busuttil RW, Tanaka K. The utility of marginal donors in liver transplantation. Liver Transpl 2003;9:651-63.

12. Barshes NR, Horwitz I, Franzini L, et al. Waitlist mortality decreases with increased use of extended criteria donor liver grafts at adult liver transplant centers. Am J Transplant 2007;7:1265-70.

13. Tector AJ, Mangus RS, Chestovich P, et al. Use of extended criteria livers decreases wait time for liver transplantation without adversely impacting posttransplant survival. Ann Surg 2006;244:439-50.

14. Álvarez-Mercado AI, Gulfo J, Romero Gómez M, et al. Use of steatotic grafts in liver transplantation: Current status. Liver Transpl 2019;25:771-86.

15. Linares I, Hamar M, Selzner N, et al. Steatosis in Liver Transplantation: Current Limitations and Future 
Strategies. Transplantation 2019;103:78-90.

16. Liu Z, Jia J, Ning H, et al. Systematic Evaluation of the Safety Threshold for Allograft Macrovesicular Steatosis in Cadaveric Liver Transplantation. Front Physiol 2019;10:429.

17. Kalisvaart M, de Haan JE, Polak WG, et al. Comparison of postoperative outcomes between donation after circulatory death and donation after brain death liver transplantation using the comprehensive complication index. Ann Surg 2017;266:772-8.

18. Rana A, Hardy M, Halazun K, et al. Survival outcomes following liver transplantation (SOFT) score: a novel method to predict patient survival following liver transplantation. Am J Transplant 2008;8:2537-46.

19. Nicolau-Raducu R, Cohen AJ, Bokhari A, et al. Predictive model and risk factors associated with a revised definition of early allograft dysfunction in liver transplant recipients. Clin Transplant 2017;31:e13097.

20. Briceño J, Padillo J, Rufián S, et al. Assignment of steatotic livers by the Mayo model for end-stage liver disease. Transpl Int 2005;18:577-83.

21. Quan H, Sundararajan V, Halfon P, et al. Coding algorithms for defining comorbidities in ICD-9-CM and ICD-10 administrative data. Med Care 2005;43:1130-9.

22. de Graaf EL, Kench J, Dilworth P, et al. Grade of deceased donor liver macrovesicular steatosis impacts graft and recipient outcomes more than the Donor Risk Index. J Gastroenterol Hepatol 2012;27:540-6.

23. Kamath PS, Kim WR. Advanced Liver Disease Study Group. The model for end-stage liver disease (MELD). Hepatology 2007;45:797-805.

24. Cholongitas E, Papatheodoridis G, Vangeli M, et al. Systematic review: the model for end-stage liver diseaseshould it replace Child-Pugh's classification for assessing prognosis in cirrhosis? Aliment Pharmacol Ther 2005;22:1079-89.

25. Orsini N, Bellocco R, Greenland SJ. Generalized least squares for trend estimation of summarized dose-response data. Stata Journal 2006;6:40-57.

26. Pencina MJ, D'Agostino RBJSim. Overall C as a measure of discrimination in survival analysis: model specific population value and confidence interval estimation. Stat Med 2004;23:2109-23.

27. Harrell FE Jr. rms: Regression modeling strategies. 2016;5.

28. Moosburner S, Sauer IM, Gassner JM, et al. Macrosteatosis is a huge problem in liver transplantation-however, not the only one we face. Am J Transplant 2019;19:2661-2.
29. Boteon YL, Boteon AP, Attard J, et al. Ex situ machine perfusion as a tool to recondition steatotic donor livers: Troublesome features of fatty livers and the role of defatting therapies. A systematic review. Am J Transplant 2018;18:2384-99.

30. Li J, Liu B, Yan LN, et al. Reversal of graft steatosis after liver transplantation: prospective study. In: Transplantation proceedings. Elsevier; 2009:3560-63.

31. Han S, Ko JS, Kwon G, et al. Effect of pure microsteatosis on transplant outcomes after living donor liver transplantation: A matched case-control study. Liver Transpl 2014;20:473-82.

32. Han S, Ha SY, Park CK, et al. Microsteatosis may not interact with macrosteatosis in living donor liver transplantation. J Hepatol 2015;62:556-62.

33. Croome KP, Lee DD, Croome S, et al. Does donor allograft Microsteatosis Matter? comparison of outcomes in liver transplantation with a propensity matched cohort. Liver Transpl 2019;25:1533-40.

34. Hoyer DP, Paul A, Gallinat A, et al. Donor information based prediction of early allograft dysfunction and outcome in liver transplantation. Liver Int 2015;35:156-63.

35. Zamboni F, Franchello A, David E, et al. Effect of macrovescicular steatosis and other donor and recipient characteristics on the outcome of liver transplantation. Clin Transplant 2001;15:53-7.

36. Kulik U, Lehner F, Klempnauer J, et al. Primary nonfunction is frequently associated with fatty liver allografts and high mortality after re-transplantation. Liver Int 2017;37:1219-28.

37. Croome KP, Lee DD, Taner CB. The "Skinny" on Assessment and Utilization of Steatotic Liver Grafts: A Systematic Review. Liver Transpl 2019;25:488-99.

38. Liu Z, Que S, Xu J, et al. Alanine aminotransferaseold biomarker and new concept: a review. Int J Med Sci 2014;11:925-35.

39. den Dulk AC, Sebib Korkmaz K, de Rooij BJ, et al. High peak alanine aminotransferase determines extra risk for nonanastomotic biliary strictures after liver transplantation with donation after circulatory death. Transpl Int 2015;28:492-501.

40. Gehrau RC, Mas VR, Dumur CI, et al. Donor hepatic steatosis induce exacerbated ischemia-reperfusion injury through activation of innate immune response molecular pathways. Transplantation 2015;99:2523-33.

41. Rana A, Petrowsky H, Hong JC, et al. Blood transfusion requirement during liver transplantation is an important risk factor for mortality. J Am Coll Surg 2013;216:902-7. 
42. Hartmann M, Szalai C, Saner FH. Hemostasis in liver transplantation: Pathophysiology, monitoring, and treatment. World J Gastroenterol 2016;22:1541-50.

43. Šeda O, Cahova M, Míková I, et al. Hepatic gene expression profiles differentiate steatotic and non-steatotic grafts in liver transplant recipients. Front Endocrinol (Lausanne) 2019;10:270.

Cite this article as: Liu Z, Wang W, Zhuang L, Liu J, Que S, Zhu D, Dong L, Yu J, Zhou L, Zheng S. Clear mortality gap caused by graft macrosteatosis in Chinese patients after cadaveric liver transplantation. HepatoBiliary Surg Nutr 2020;9(6):739-758. doi: 10.21037/hbsn.2019.12.02
44. Raza A, Dikdan G, Desai KK, et al. Global gene expression profiles of ischemic preconditioning in deceased donor liver transplantation. Liver Transpl 2010;16:588-99.

45. Lee S, Zhang C, Liu Z, et al. Network analyses identify liver-specific targets for treating liver diseases. Mol Syst Biol 2017;13:938. 